\title{
Identifying uncertainty contributions to the seismic fragility assessment of a nuclear reactor steam line
}

\author{
Pierre Gehl $^{1 *}$, Marine Marcilhac-Fradin ${ }^{2}$, Jeremy Rohmer $^{1}$, Yves Guigueno ${ }^{2}$, Nadia Rahni ${ }^{2}$, Julien \\ Clément $^{2}$ \\ ${ }^{1}$ BRGM \\ Orléans, France \\ p.gehl@brgm.fr, j.rohmer@brgm.fr \\ ${ }^{2}$ IRSN \\ Fontenay-aux-Roses, France \\ marine.marcilhacfradin@irsn.fr, yves.guigueno@irsn.fr, nadia.rahni@irsn.fr, julien.clement@irsn.fr
}

\begin{abstract}
In nuclear applications, fragility curves are an essential element of the seismic probabilistic safety assessment that is performed at the level of the power plant. These statistical tools establish the link between the probabilistic seismic hazard loading at the site and the required performance of the plant's safety functions. In this context, the fragility model formalized by Kennedy \& Ravindra (1984) proposes to account for the aleatory randomness and the epistemic uncertainty generated by various sources of variability, such as the representation of the seismic input or the assumptions in the structural model and in the loading patterns.
\end{abstract}

Therefore, this study investigates the relative contributions of such variables to the dispersion of the resulting fragility functions, while ensuring the separation between aleatory and epistemic uncertainty sources, as advocated by the standards in effect in the nuclear industry. To this end, a coupled model of a supporting structure and an anchored steam line is considered and computed with the finite-element CAST3M software (Rahni et al., 2017). The failure criterion is related to the exceedance of a given effort value at a point of the steam line corresponding to a vertical stop. A coupled of hundreds of threecomponent ground-motion records are applied at the base of the model, in order to perform non-linear timehistory analyses. The record selection follows the conditional spectrum method (Lin et al., 2013), in order to scale the records while ensuring the hazard consistency, through the identification of reference earthquake scenarios for each predefined hazard level.

As a result, dozens of ground-motion parameters are tested as potential intensity measures (IMs), with respect to state-of-the-art indicators measuring efficiency or sufficiency. Some couples of ground-motion parameters are assembled as vector-valued IMs, in order to generate so-called fragility surfaces: these multivariate functions may then be reinterpreted as single-IM fragility curves, where the conditional distribution of the secondary IM with respect to the main one generates additional confidence intervals for the fragility curve. It is thus shown that the aleatory randomness due to record-to-record variability may be partially transferred to an epistemic uncertainty contribution. This variability is then put in perspective with other sources of uncertainty due to modelling assumptions, such as the variability in the mechanical parameters of the model or the angle of loading of the seismic input.

\section{References}

[1] Kennedy, R.P., \& Ravindra, M.K. (1984). Seismic fragilities for nuclear power plant studies. Nuclear Engineering and Design, 79(1), 47-68.

[2] Lin, T., Haselton, C., \& Baker, J.W. (2013). Conditional spectrum-based ground motion selection - Part I. Earthquake Engineering and Structural Dynamics, 42(12), 1847-1865.

[3] Rahni, N., et al. (2017). An original approach to derived seismic fragility curves - Application to a PWR main steam line. In Proceedings of the International Topical Meeting on Probabilistic Safety Assessment and Analysis (PSA2017), Pittsburgh, PA. 